\title{
DIFFUSING CAPACITY PREDICTS OPERATIVE MORTALITY BUT NOT LONG-TERM SURVIVAL AFTER RESECTION FOR LUNG CANCER
}

Jun Wang, MD

Jemi Olak, MD

Mark K. Ferguson, MD
Objectives: We sought to determine whether diffusing capacity influences operative mortality and long-term survival after resection for lung cancer. Methods: We retrospectively reviewed the case histories of patients who underwent major resection for lung cancer. The association between operative mortality and predicted postoperative diffusing capacity was examined. Long-term survival among operative survivors was compared between the groups with high and low predicted postoperative diffusing capacity. Results: The group comprised 410 patients with a mean age of 62.3 years. We performed 273 lobectomies, 35 bilobectomies, and 102 pneumonectomies. A total of 32 operative deaths (7.8\%) were associated with low predicted postoperative diffusing capacity $(P<.001)$. If we examine only operative survivors, there is no significant difference in survival data between patients with a predicted postoperative diffusing capacity of less than 50 and those with a predicted figure of 50 or more (stage I, 111 vs 90 months; stage II, 26 vs 32 months; stage IIIa 32 vs 26 months; $\log$ rank $P>.5$ for each). On the basis of the Cox proportional hazards model, predicted postoperative diffusing capacity did not have a statistically significant effect on longterm survival (estimated hazard ratio corresponding to a 20-point decrease in predicted postoperative diffusing capacity $=1.13 ; 95 \%$ confidence interval: 0.92 to 1.37$)$. Conclusion: A poor diffusing capacity is associated with high operative mortality but does not adversely affect long-term survival after major lung resection among operative survivors. Improving the perioperative management of patients undergoing major lung resection may enable inclusion of more patients with reduced diffusing capacity in the candidate pool for surgery, thus maximizing survival for early-stage lung cancer. (J Thorac Cardiovasc Surg 1999;117:581-7)
Tisions he appropriate selection of patients with lung cancer for major lung resection is a continuing challenge. The primary focus has been on reducing surgical mortality and complications, which for many years has been accomplished by the use of a general assessment of the patient and measurement of spirometric parame-

From the Section of Thoracic Surgery, Department of Surgery, The University of Chicago, Chicago, Ill.

Read at the Seventy-eighth Annual Meeting of The American Association for Thoracic Surgery, Boston, Mass, May 3-6, 1998.

Received for publication May 8, 1998; revisions requested July 6, 1998; revisions received Oct 28, 1998; accepted for publication Oct 28, 1998.

Address for reprints: Mark K. Ferguson, MD, 5841 S Maryland Ave, MC5035, Chicago, IL 60637.

Copyright (C) 1999 by Mosby, Inc.

$0022-5223 / 99 \$ 8.00+0 \quad \mathbf{1 2 / 6 / 9 5 5 9 8}$ ters. In addition, diffusing capacity, exercise capacity, and oxygen consumption during exercise have recently been shown to be important independent predictors of operative mortality and pulmonary morbidity. ${ }^{1-6}$

The focus of patient assessment has recently expanded to include long-term outcomes. Although tumor stage has always been taken into account in such an assessment, physiologic parameters have not been used extensively in this regard. It has recently been shown that diffusing capacity predicts intermediate-term morbidity in patients undergoing major resection for lung cancer. ${ }^{7}$ In addition, diffusing capacity has been shown to be a predictor of long-term survival in patients with severe emphysema. ${ }^{8-10}$ Patients with lung cancer have a diminished diffusing capacity compared with the general population, but whether diffusing capacity influences long-term outcome after major lung resection is un- 


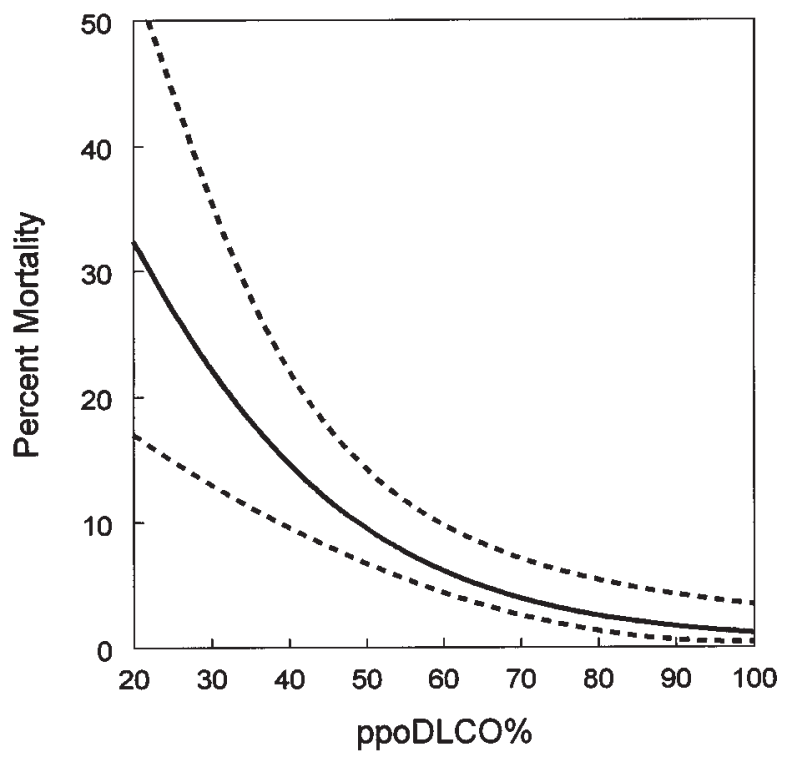

Fig 1. Estimated operative mortality after major pulmonary resection for non-small cell lung cancer as a function of the predicted postoperative diffusing capacity expressed as a percent of predicted (ppoDLCO\%) for 334 patients. Solid line, Estimated from logistic regression model; dashed lines, $95 \%$ confidence limits.

known. ${ }^{11}$ We performed a retrospective analysis of long-term survival in patients who have undergone major resection for lung cancer to address this issue.

\section{Patients and methods}

We performed a retrospective analysis of patients who underwent major lung resection (lobectomy, bilobectomy, or pneumonectomy) at the University of Chicago from January 1980 through June 1997. Data were recorded for preoperative and operative risk factors as previously described. ${ }^{5}$ Spirometry and measurement of total lung capacity and single-breath diffusing capacity for carbon monoxide were performed as previously described. ${ }^{2}$ Predicted postoperative diffusing capacity (ppoDLCO\%) was calculated by multiplying the percent of predicted normal for each patient by the fraction of functional lung segments remaining after resection. Functional lung segments were assigned as described previously. ${ }^{5}$

Patients were staged according to the American Joint Commission on Cancer ${ }^{12}$ staging system. Data on postoperative morbidity and mortality were recorded and operative mortality was defined as death during hospitalization for the operation or within 30 days of the operation. The relationship between ppoDLCO $\%$ and operative mortality was examined by fitting a stepwise regression model. Candidate covariates in addition to ppoDLCO $\%$ were age, sex, performance status, type of operation, stage, and pathology (squamous cell cancer, adenocarcinoma, or other).

Survival was calculated from the day of operation by means of the Kaplan-Meier method. ${ }^{13}$ For the analysis of all

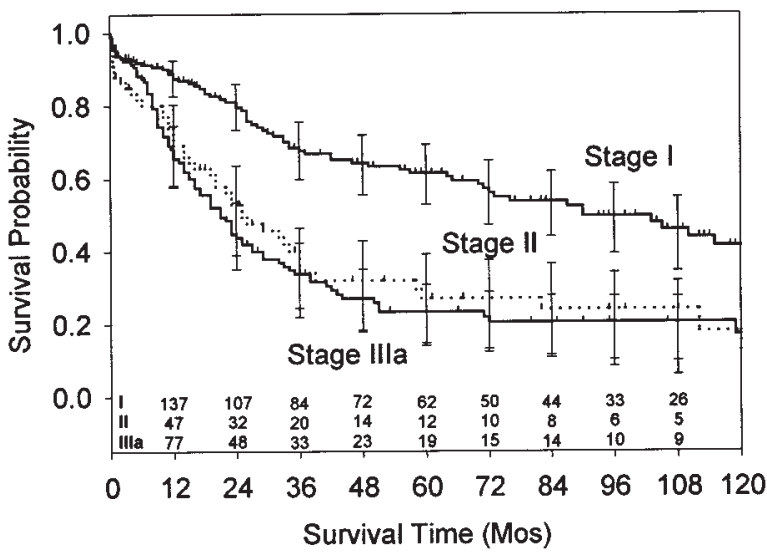

Fig 2. Kaplan-Meier survival curves by stage after major pulmonary resection for non-small cell lung cancer. Vertical hash marks represent censored values. Error bars depict $95 \%$ confidence limits $(P<.001)$. The number of deaths was 71, 46 , and 89 in stage I, stage II, and stage IIIa, respectively.

patients and of operative survivors only, based on previously published calculations regarding risk of mortality and its relationship to ppoDLCO \%, patients were divided into two groups with ppoDLCO $\%=50$ as a cutoff point. The groups were compared by means of the log rank test with stratification according to stage. ${ }^{5,14}$ The relationship between ppoDLCO \% and long-term survival was also examined with the Cox proportional hazards model, treating ppoDLCO $\%$ as a continuous variable and adjusting for age, sex, performance status, operation type, stage, and pathology, by means of a backward elimination procedure. ${ }^{15}$ Inspection of Martingale residuals indicated that the untransformed values of ppoDLCO $\%$ and age provided an adequately fitting model. ${ }^{16}$ The proportional hazards assumption was checked by including interaction terms between each covariate and (log) survival time, as well as through examination of Schoenfeld residuals. ${ }^{17}$ None of the interaction terms was statistically significant, and the residual plots indicated that the proportional hazards assumption was reasonable. All statistical calculations were performed with Minitab 10.5 (Minitab, Inc, State College, Pa), Systat 7.0 (SPSS, Inc, Chicago, Ill), or SAS (SAS Institute, Inc, Cary, NC) software. Data are expressed as mean \pm standard deviation.

\section{Results}

The group comprised 410 patients (242 men, 168 women) with a mean age of $62.3 \pm 9.9$ years (range 35 87 years). Included were 196 patients with squamous cell cancer, 186 with adenocarcinoma, and 14 each with large cell cancer or bronchoalveolar carcinoma. The mean preoperative diffusing capacity expressed as a percent of predicted (available in 334 patients; data were missing for the remaining patients) was $85.9 \pm$ 21.9 (range 21-171) and the mean ppoDLCO\% was $61.4 \pm 18.8$ (range 11.1-132.3; 334 patients). 

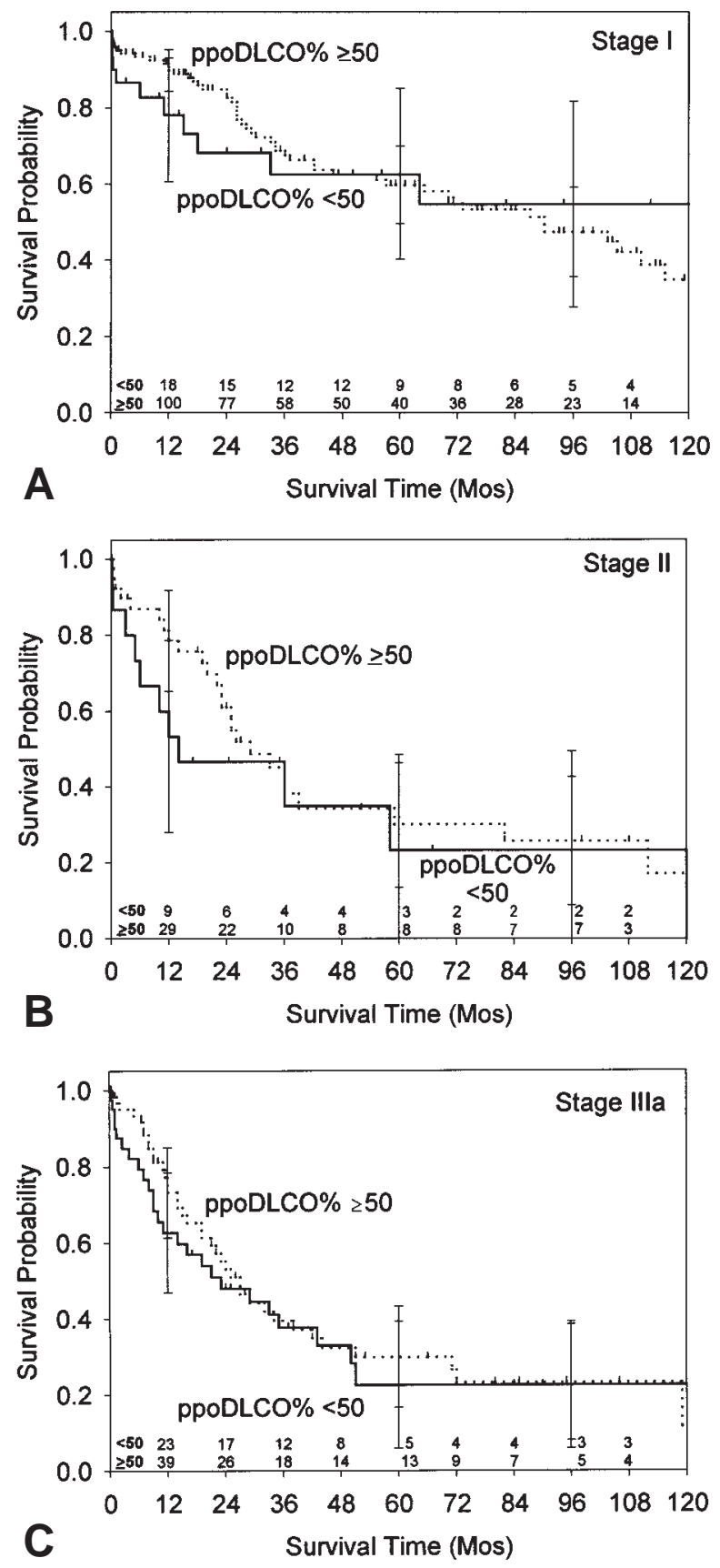

Fig 3. A to C, Kaplan-Meier survival curves by stage after major pulmonary resection for non-small cell lung cancer. Patients with a predicted postoperative diffusing capacity expressed as a percent of predicted ( $p p o D L C O \%$ ) of $<50$ are compared with patients with a ppoDLCO $\% \geq 50$. Vertical hash marks represent censored values. Error bars represent 95\% confidence limits.

Patients in whom diffusing capacity was not measured were more likely to have been operated on during the first 6 years of the study period compared with
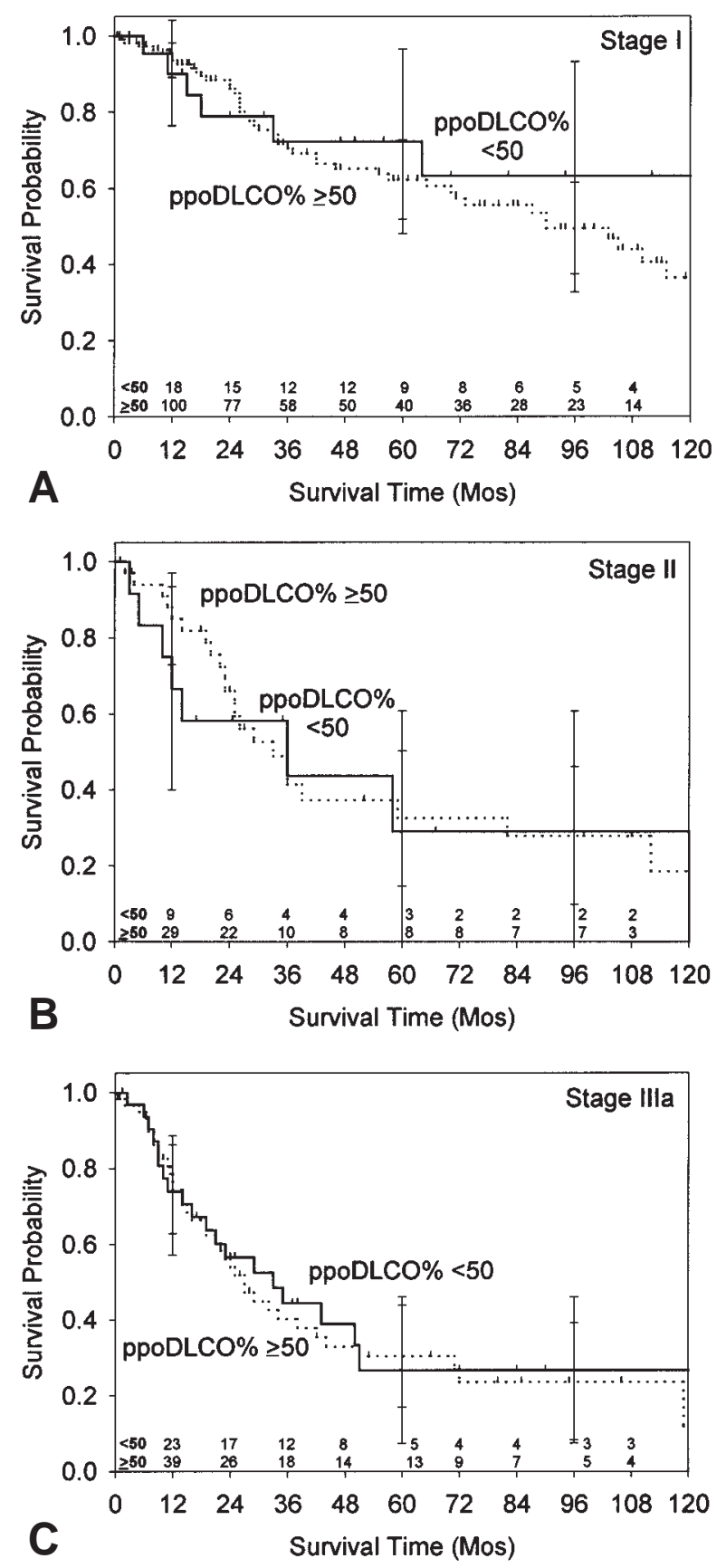

Fig 4. A to C, Kaplan-Meier survival curves by stage for operative survivors after major pulmonary resection for non-small cell lung cancer. Patients with a predicted postoperative diffusing capacity expressed as a percent of predicted (ppoDLCO\%) of < 50 are compared with patients with a ppoDLCO $\% \geq 50$. Vertical hash marks represent censored values. Error bars represent $95 \%$ confidence limits.

either of the latter 6-year intervals $\left(P<.001\right.$ by $\chi^{2}$ analysis). In addition, they had lower forced vital capacity $(2.89 \pm 0.11$ vs $3.28 \pm 0.05 \mathrm{~L} ; P=.002)$, a 
Table I. Postoperative predicted DLCO values for patients by risk group and stage

\begin{tabular}{|c|c|c|c|c|c|}
\hline & Stage I & Stage II & Stage IIIa & Stages IIIb, IV & All stages \\
\hline \multicolumn{6}{|l|}{$\operatorname{ppoDLCO} \%<50$} \\
\hline Patients & 30 & 15 & 43 & 5 & 93 \\
\hline ppoDLCO $\%$ & $41.9 \pm 5.4$ & $39.6 \pm 7.6$ & $37.2 \pm 8.7$ & $42.2 \pm 8.1$ & $39.3 \pm 7.7$ \\
\hline \multicolumn{6}{|l|}{ ppoDLCO $\%>50$} \\
\hline Patients & 127 & 40 & 67 & 5 & 239 \\
\hline ppoDLCO $\%$ & $71.9 \pm 15.3$ & $69.3 \pm 13.4$ & $67.2 \pm 12.5$ & $68.3 \pm 8.3$ & $70.0 \pm 14.2$ \\
\hline \multicolumn{6}{|l|}{ All patients } \\
\hline Patients & 157 & 55 & 110 & 10 & $332 *$ \\
\hline ppoDLCO $\%$ & $66.1 \pm 18.3$ & $61.2 \pm 18.0$ & $55.5 \pm 18.5$ & $55.3 \pm 15.8$ & $61.4 \pm 18.8$ \\
\hline
\end{tabular}

ppoDLCO\%, Calculated postoperative diffusing capacity expressed as a percent of predicted.

*Cell type for two patients was not known.

lower forced vital capacity expressed as a percent of predicted $(77.7 \% \pm 3.2 \%$ vs $87.1 \% \pm 0.9 \% ; P=.006)$, and a lower forced expiratory volume in 1 second $\left(\mathrm{FEV}_{1} ; 2.06 \pm 0.08\right.$ vs $\left.2.25 \pm 0.04 \mathrm{~L} ; P=.036\right)$ than did the patients who had diffusing capacity measured. The two groups of patients were not significantly different in terms of age, sex, $\mathrm{FEV}_{1}$ expressed as a percent of predicted, type of operation, cancer cell type, or pathologic stage.

Lobectomy was performed in 273 patients, bilobectomy in 35, and pneumonectomy in 102 patients. The pathologic stage was I in 184 patients, II in 68, IIIa in 143 , IIIb in 8 , IV in 4 patients, and was unknown in 3 patients. The mean postoperative length of stay was $11.6 \pm 10.0$ days (range 0-120 days), which decreased linearly from $14.5 \pm 8.1$ days during the years 1980 to 1984 to $8.3 \pm 10.4$ days for operations performed since January 1995 . The mortality rate was $7.8 \% \quad(32$ patients), which also decreased linearly from $8.8 \%$ (13/148) during the years 1980 to 1984 to $5.6 \%(4 / 71)$ for operations performed since January 1995. The operative mortality rate was similar for patients who had diffusing capacity measured and for those who did not (7.4\% vs $9.2 \% ; P=.61)$. The mortality rate was closely related to the ppoDLCO\% (Fig 1). The unadjusted odds ratio corresponding to a 20-point decrease in ppoDLCO\% was 2.7 (95\% CI: 1.6-4.6). From the multivariable logistic regression analysis, the odds of death are estimated to increase 3.5-fold (95\% CI: 1.9-6.2) for every 20-point decrease in ppoDLCO $\%$ after adjustment for patient age (age was the only significant predictor $[P=.014]$ among the remaining covariates).

The mean follow-up was 37.2 months (range 0-214 months). Survival by stage for all patients is shown in Fig 2. Survival by stage for all patients with diffusing capacity measured and separating patients into higherrisk and lower-risk ppoDLCO\% groups demonstrated no statistically significant difference in survival be- tween the two groups within each stage (stages I, II, and IIIa only; 322 patients; Fig 3; $\log \operatorname{rank} P>.25$ for each). Median survival times for patients with ppoDLCO $\%<50$ and ppoDLCO $\% \geq 50$ were as follows: stage I, 83.8 versus 88.1 months; stage II, 13.0 versus 27.8 months; stage IIIa, 21.7 versus 25.4 months. Median survival times for patients surviving surgery with diffusing capacity measured and separating patients into higher-risk and lower-risk ppoDLCO\% groups (ppoDLCO $\%<50$ and ppoDLCO $\% \geq 50$ ) were as follows: stage I, 111.3 versus 89.6 months; stage II, 26.5 versus 31.9 months; stage IIIa, 31.6 versus 25.8 months. No difference in survival was detected between the two groups within each stage (Fig 4; log rank > 0.5 for each).

Using ppoDLCO\% as a continuous variable in a multivariable Cox proportional hazards model, we also found no statistically significant effect of ppoDLCO\% on long-term survival. The relative death rate for a 20 point decrease in ppoDLCO\% was 1.13 (95\% CI 0.921.37 ) and for a 30-point decrease in ppoDLCO $\%, 1.19$ (95\% CI 0.89-1.61). The only significant predictors of increased mortality were more advanced pathologic stage $(P<.001)$ and increased age at operation (relative death rate per 10 -year increase in age $=1.31 ; 95 \% \mathrm{CI}$ $1.09-1.58, P=.003)$.

\section{Discussion}

The traditional assessment of patients with lung cancer who are candidates for major lung resection has focused primarily on estimating the risk of operative mortality and perioperative morbidity. This has been accomplished by means of a general evaluation of age and performance status, measurement of spirometric values, and, recently, evaluation of gas exchange parameters such as diffusing capacity or a measurement of exercise capacity or oxygen consumption during exercise. $^{1-6}$ The long-term outcome after operation has been 
estimated primarily on the basis of patient age, general performance status, and pathologic stage. Using a combination of all of these factors, surgeons make important judgments regarding operability in individual patients.

During the past several years a trend has developed that emphasizes the inclusion of intermediate- and long-term outcome expectations in the initial assessment of the candidate for major lung resection. Age has been shown to be a factor that determines operative mortality and early postoperative morbidity, as was found in our study. ${ }^{5,18,19}$ Evaluating operative survivors only, the long-term survival by stage for older compared with younger patients has been shown to be similar. $^{20-23}$ However, we detected a highly significant effect of age on long-term survival. Similarly, a low diffusing capacity predicts a higher incidence of postoperative pulmonary complications and, in some reports, operative mortality. ${ }^{1,2,5,7}$ A low diffusing capacity predicts a higher likelihood of pulmonary complications in the intermediate term, which require either supplemental oxygen or hospitalization, and a worse dyspnea score. Patients with a lower diffusing capacity also may have a worse long-term survival than those with a normal diffusing capacity. ${ }^{7}$

The focus on diffusing capacity is appropriate. Patients with lung cancer have lower diffusing capacities than patients without lung cancer who have similar spirometric values and a similar smoking history, suggesting that patients with lung cancer have more severe but still subclinical emphysema. ${ }^{11}$ Diffusing capacity is inversely related to the mortality rate in patients with severe emphysema. ${ }^{1,2,5}$ On the basis of these findings, we thought it appropriate to investigate the relationship of diffusing capacity to long-term survival in patients undergoing major lung resection for lung cancer.

We found that in our series of patients the ppoDLCO\% was strongly associated with the risk of operative mortality, confirming previous reports by us and others. ${ }^{1,2,5}$ However, in patients who survived major lung resection, the ppoDLCO $\%$ did not have a statistically significant influence on long-term survival after adjusting for stage of disease and age at the time of operation. The reasons for this discrepancy are not immediately clear. Patients who undergo major lung resection for cancer have higher diffusing capacities, on average, than patients with end-stage emphysema, in whom a low diffusing capacity is associated with a decreased long-term survival. This may explain why patients with lung cancer avoid the detrimental influence of diffusing capacity on long-term survival. It is also possible that the increased operative mortality associated with a low diffusing capacity in the patients undergoing lung resection skews the survival curves by eliminating patients who would have a poorer longterm survival on the basis of their diffusing capacity. Were these patients to survive an operation, a detrimental effect of low diffusing capacity on subsequent survival might become apparent. However, recent reports suggest that long-term survival in some patients with severe underlying lung disease may actually be improved with lung resection, mirroring some of the effects seen with lung volume reduction surgery in patients with severe emphysema. ${ }^{24-29}$ In any case, on the basis of the upper confidence limits, an increase in the death rate of as much as $37 \%$ for a 20 -point reduction in diffusing capacity and an increase in the death rate of as much as $61 \%$ for a 30-point reduction in diffusing capacity are consistent with our data. Thus larger studies are needed to confirm our results.

The finding of a low ppoDLCO $\%$ in a patient has been interpreted by some physicians to mean that the patient is not a candidate for major lung resection. However, the use of ppoDLCO\% has not been proffered as an absolute measure of a patient's ability to withstand surgery, but merely as one means, albeit an important one, to assess relative risk of operative morbidity and mortality. In fact, a number of improvements in intraoperative and postoperative management of patients with compromised pulmonary status have been developed in recent years in conjunction with the increasing clinical experience in lung transplantation and lung volume reduction surgery for emphysema. These advances will likely reduce the risk of operative morbidity and mortality of major lung resection over time, possibly reducing the negative influence that ppoDLCO $\%$ has on these outcomes.

Resection remains the optimal therapy for early-stage lung cancers. It is important that surgical therapy be offered to as many patients with early-stage lung cancer as possible, commensurate with their risk of perioperative complications and the expected benefit to them in long-term survival. It is incumbent on surgeons and other physicians to further improve the current techniques of perioperative management so that patients who are at higher operative risk on the basis of a marginal ppoDLCO $\%$ can be safely included in the candidate pool for surgery. Assuming that operating on more patients with reduced diffusing capacity would not adversely affect long-term survival figures, survival in the patient with early-stage lung cancer should improve.

We gratefully acknowledge the assistance of Theodore Karrison, $\mathrm{PhD}$, for assisting in the statistical analyses. 


\section{REFERENCES}

1. Markos J, Mullan BP, Hillman DR, Musk AW, Antico VF, Lovegrove FT, et al. Preoperative assessment as a predictor of mortality and morbidity after lung resection. Am Rev Respir Dis 1989;139:902-10.

2. Ferguson MK, Little L, Rizzo L, Popovich KJ, Glonek GF, Leff A, et al. Diffusing capacity predicts morbidity and mortality after pulmonary resection. J Thorac Cardiovasc Surg 1988;96:894900.

3. Walsh GL, Morice RC, Putnam JB Jr, Nesbitt JC, McMurtrey MJ, Ryan MB, et al. Resection of lung cancer is justified in high-risk patients selected by exercise oxygen consumption. Ann Thorac Surg 1994;58:704-11.

4. Bolliger CT, Jordan P, Soler M, Stulz P, Gradel E, Skarvan K, et al. Exercise capacity as a predictor of postoperative complications in lung resection candidates. Am J Respir Crit Care Med 1995;151:1472-80.

5. Ferguson MK, Reeder LB, Mick R. Optimizing selection of patients for major lung resection. J Thorac Cardiovasc Surg 1995; 109:275-83.

6. Pate P, Tenholder MF, Griffin JP, Eastridge CE, Weiman DS. Preoperative assessment of the high-risk patient for lung resection. Ann Thorac Surg 1996;61:1494-500.

7. Bousamra M II, Presberg KW, Chammas JH, Tweddell JS, Winton BL, Bielefeld MR, et al. Early and late morbidity in patients undergoing pulmonary resection with low diffusion capacity. Ann Thorac Surg 1996;62:968-75.

8. Kanner RE, Renzetti AD Jr, Stanish WM, Barkman HW Jr, Klauber MR. Predictors of survival in subjects with chronic airflow limitation. Am J Med 1983;74:249-55.

9. Anthonisen NR. Prognosis in chronic obstructive pulmonary disease: results from multicenter clinical trials. Am Rev Respir Dis 1989;140:S95-9.

10. Naunheim KS, Ferguson MK. The current status of lung volume reduction operations for emphysema. Ann Thorac Surg 1996;62:601-12.

11. Menna Barretto SS, McClean PA, Szalai JP, Zamel N. Reduction of lung diffusion for carbon monoxide in patients with lung carcinoma. Chest 1993;103:1142-6.

12. American Joint Committee on Cancer. Lung. In: Beahrs $\mathrm{OH}$, Henson DE, Hutter RVP, Kennedy BJ, editors. Manual for staging of cancer. 4th ed. Philadelphia: JB Lippincott; 1992. p. 115-9.

13. Kaplan EL, Meier P. Nonparametric estimation from incomplete observations. J Am Stat Assoc 1958;53:457-81.

14. Peto R, Pike MC, Armitage P, Breslow NE, Cox DR, Howard SV, et al. Design and analysis of randomized clinical trials requiring prolonged observation of each patient. Br J Cancer 1977;35:1-39.

15. Cox DR. Regression models and life tables. J R Stat Soc Series B 1972;34:187-220.

16. Fleming TR, Harrington DP. Counting processes and survival analysis, sec 4.6. New York: John Wiley; 1991.

17. Schoenfeld D. Partial residuals for the proportional hazards regression model. Biometrika 1982;69:239-41.

18. Ginsberg RJ, Hill LD, Eagan RT, Thomas P, Mountain CF, Deslauriers J, et al. Modern thirty-day operative mortality for surgical resections in lung cancer. J Thorac Cardiovasc Surg 1983; 86:654-8

19. Kohman LJ, Meyer JA, Ikins PM, Oates RP. Random versus predictable risks of mortality after thoracotomy for lung cancer. J Thorac Cardiovasc Surg 1986;91:551-4.
20. Sherman S, Guidot CE. The feasibility of thoracotomy for lung cancer in the elderly. JAMA 1987;258:927-30.

21. Roxburgh JC, Thompson J, Goldstraw P. Hospital mortality and long-term survival after pulmonary resection in the elderly. Ann Thorac Surg 1991;51:800-3.

22. Mizushima Y, Noto H, Sugiyama S, Kusajima Y, Yamashita R, Kashii T, et al. Survival and prognosis after pneumonectomy for lung cancer in the elderly. Ann Thorac Surg 1997;64:193-8.

23. Pagni S, Federico JA, Ponn RB. Pulmonary resection for lung cancer in octogenarians. Ann Thorac Surg 1997;63:785-9.

24. Cerfolio RJ, Allen MS, Trastek VF, Deschamps C, Scanlon PD, Pairolero PC. Lung resection in patients with compromised pulmonary function. Ann Thorac Surg 1996;62:348-51.

25. McKenna RJ Jr, Fischel RJ, Brenner M, Gelb AF. Combined operations for lung volume reduction surgery and lung cancer. Chest 1996;110:885-8.

26. Cordova F, O’Brien G, Furukawa S, Kuzma AM, Travaline J, Criner GJ. Stability of improvements in exercise performance and quality of life following bilateral lung volume reduction surgery in severe COPD. Chest 1997;112:907-15.

27. Hazelrigg SR, Boley TM, Weber D, Magee MJ, Naunheim KS. Incidence of lung nodules found in patients undergoing lung volume reduction. Ann Thorac Surg 1997;64:303-6.

28. Ojo TC, Martinez F, Paine R III, Christensen PJ, Curtis JL, Weg $\mathrm{JG}$, et al. Lung volume reduction surgery alters management of pulmonary nodules in patients with severe COPD. Chest 1997;112:1494-500.

29. DeRose JJ Jr, Argenziano M, El-Amir N, Jellen PA, Gorenstein LA, Steinglass KM, et al. Lung reduction operation and resection of pulmonary nodules in patients with severe emphysema. Ann Thorac Surg 1998;65:314-8.

\section{Discussion}

Dr Joseph I. Miller, Jr (Atlanta, Ga). I congratulate Dr Jun Wang and his coauthors on an excellent manuscript. Dr Wang is the 1997-1998 Evarts A. Graham Fellow, and this presentation is one of his many accomplishments during his year in the United States.

This paper marks the completion of a trilogy for Dr Ferguson and his colleagues from the University of Chicago. In 1988 Dr Ferguson presented a paper before the Association entitled "Diffusing Capacity Predicts Mortality and Morbidity After Pulmonary Resection.” In 1994 he presented before the Association a paper entitled "Optimizing Selection of Patients for Major Lung Resection," at which time he first introduced the concept of ppoDLCO\%. He also pointed out at that time that this was the most specific indicator of postoperative morbidity and mortality, but there was also no correlation between it and use of percent predicted $\mathrm{FEV}_{1}$ as a postoperative indicator. This current paper marks the completion of an outstanding series of investigative and statistical studies on diffusing capacity and ppoDLCO\%.

I have several questions. In this excellent statistical study, the ppoDLCO\% correlates well with early morbidity and mortality but is not an indicator of long-term survival. In making this assumption, you have taken out the cohort of patients dying early, that is, 32 patients, or $7.2 \%$ of the total series that were stated to be operative deaths. Do you think 
there would have been any difference in the statistics had this operative cohort of 32 patients been included with your analysis?

From studies on chronic obstructive pulmonary disease, we know that an $\mathrm{FEV}_{1}$ of less than $30 \%$ of predicted or a diffusing capacity of less than $30 \%$ of predicted correlates well with a 5-year survival of less than 50\%. In the era of lung volume surgery, we know that the lower the diffusing capacity, the greater the risk of morbidity and mortality. The current study correctly points out that a low diffusing capacity should not be the only criterion for operability; rather, a number of parameters should be evaluated.

My questions, then, are these: Do you think that if the operative cohort of 37 patients had been left in the total group the statistics would have been different? Do you use oxygen consumption as an indicator of operability in your marginal cases? What parameter other than ppoDLCO\% do you think is helpful in deciding resectability in marginal cases? In patients in the marginal group whom you are considering for pulmonary resection, patients who have both low diffusing capacities and low flow rates, do you think a right heart catheterization would ever be indicated to rule out pulmonary hypertension?

Your mean diffusing capacity was $85.9 \%$, or $86 \% \pm 21 \%$, and the mean ppoDLCO $\%$ was 61 . This is a much higher diffusing capacity than we usually see in our patients with lung cancer. In general, our mean preoperative diffusing capacity would be in the range of $60 \%$ to $70 \%$ of predicted. There seems to be a discrepancy in the patient population between Chicago and Atlanta.

In your manuscript, you state that patients are divided into four risk groups according to their ppoDLCO\%. I did not see that list given today. Can you tell us what these divisions are and what the risks are within each group?

Dr Wang. Dr Miller, thank you for your kind comment and your many good questions.

You questioned the use of oxygen consumption as a predictor of risk. Our previous study, presented at the meeting of the American College of Chest Physicians in 1997, showed that the diffusing capacity predicted postoperative morbidity better than oxygen consumption did. Other parameters that we used to assess risk included $\mathrm{FEV}_{1}$, age, and general performance status.

Regarding right heart catheterization, we do not often see these kinds of problems. If pulmonary hypertension is suspected, the initial evaluation is echocardiography. If the pressure is abnormal, right heart catheterization is the preferred procedure.

As for your question about the mean diffusing capacity, in our study, $25 \%$ of patients had a ppoDLCO $\%$ of less than 50 . There may be a difference between the institutions in patient population.

The four risk groups identified in assessing the risk of operative mortality relative to the ppoDLCO $\%$ were those with a ppoDLCO $\%$ less than 40,40 to 50,50 to 60 , and greater than 60 .

We included the operative deaths to determine in an isolated way how ppoDLCO\% might influence long-term survival, assuming the patients survived the operation. Because ppoDLCO $\%$ predicts operative mortality, there was a difference between the groups in long-term survival when operative deaths were included in the analysis.

Age is an important determinant of operative mortality but does not appear to have much influence on long-term survival in operative survivors. Similarly, the ppoDLCO\% influences operative mortality but not long-term survival. It is our job to improve preoperative management to reduce the predictable operative mortality.

Dr Larry R. Kaiser (Philadelphia, Pa). I noted that $25 \%$ of the resections were pneumonectomies and another $8 \%$ or $9 \%$ were bilobectomies. Thus almost $35 \%$ of the patients had procedures that are associated with a higher mortality.

Is your ppoDLCO \% based on the operation you think the patient is going to have, is the prediction done prospectively, or is this postoperatively predicted after the resection is done? Did the number of pneumonectomies, which is a little high, explain the survival difference?

Dr Ferguson. The percentage of extended resections is somewhat higher than most investigators have reported, but it did not appear to influence long-term outcome in our patients. Most of these numbers were not measured. They were calculated on the basis of the type of resection that was performed. 